\title{
Spin fluctuations and the "strange metal" behavior of a weakly doped antiferromagnet
}

\author{
V.M.Loktev ${ }^{1}$, V.Turkowski ${ }^{2 \dagger}$ \\ ${ }^{1}$ Bogolyubov Institute for Theoretical Physics, Metrologichna Str. 14-b, Kyiv, 03680 Ukraine \\ 2 Department of Physics and Astronomy, University of Missouri, Columbia, Missouri 65211, USA
}

Received May 16, 2008, in final form June 18, 2008

\begin{abstract}
We analyze the spectral properties of a phenomenological model for a weakly doped two-dimensional antiferromagnet, in which the carriers move within one of the two sublattices where they were introduced. Such a constraint results in the free carrier spectra with the maxima at $\mathbf{k}=( \pm \pi / 2, \pm \pi / 2)$ observed in some cuprates. We consider the spectral properties of the model by taking into account fluctuations of the spins in the antiferromagnetic background. We show that such fluctuations lead to a non-pole-like structure of the single-hole Green's function and these fluctuations can be responsible for some anomalous "strange metal" properties of underdoped cuprates in the nonsuperconducting regime.
\end{abstract}

Key words: cuprate superconductors, underdoped regime, pseudogap

PACS: $74.20 .-z, 74.20 . F g, 74.20 . R p, 74.72 .-h$

\section{Introduction}

After more than twenty years from its discovery, the problem of high-temperature superconductivity (HTSC) remains unresolved. Nevertheless, there are some facts about HTSCs, which are generally accepted by the scientific community. In particular, it is well known that these materials transform from antiferromagnetic insulators into superconductors with the carrier doping. The superconductivity in most of the cuprates takes place mainly in the $\mathrm{CuO}_{2}$ layers, and the other inter-layer atoms supply the carriers for these layers and play a role of the carrier scatterers. It is believed that the presence of antiferromagnetic background strongly affects the physical behavior of weakly doped materials. In particular, this behavior can be defined by strong hole or electron correlations [1,2]. Moreover, the correlations can be responsible for the $d$-wave superconductivity in many cuprates $[3,4]$. In fact, since the isotope effect in optimally doped HTSCs is rather weak, it suggests that the electron-phonon coupling is not the main source of superconductivity in these materials, though the role of phonons and, in particular, the interplay of strong correlations and phonon coupling in HTSCs is currently an active area of research (see, for example, [5] and references therein). Thus, it is believed by a great part of the researchers that the phenomenon of HTSC can be explained by using a strongly correlated model, in which the superconducting pairing with the anisotropic order parameter is caused by an antiferromagnetic spin-wave coupling. Probably, the most popular models for this scenario are the two-dimensional Hubbard model and its approximation in the case of strong correlations, the $t J$-model [6,7] (see, e.g., [8-11] and [12-14], where the quasi-particle spectrum of cuprates was studied within the framework of this model).

Unfortunately, these models cannot be solved exactly in the two-dimensional case, so it is difficult to make a firm conclusion whether they can be considered as realistic models of HTSCs. As an alternative approach, one can consider a simplified phenomenological model of cuprates with strong correlations, which takes into account their main properties and can be solved exactly. Typical unusual and important properties of cuprates include a universal dome shape of the critical

\footnotetext{
*E-mail: vloktev@bitp.kiev.ua

${ }^{\dagger}$ E-mail: turkowskiv@missouri.edu
} 
temperature - doping curve, a different from the BCS theory ratio $2 \Delta(T=0) / T_{\mathrm{c}}^{\max } \simeq 5.5$ at optimal doping, different critical densities for the superconducting gap in the nodal and antinodal directions. Probably, the most unusual phenomenon in HTSC is the pseudogap phase in the underdoped regime. In this phase, the materials demonstrate very unusual properties of a "strange metal", like an anomalous temperature dependence of resistivity, etc., which are different from a Fermi-liquid behavior. There is no general agreement in the HTSC's community on the origin of this phenomena. However, a recent improvement of the angle-resolved photoemission spectroscopy(ARPES) technique gives a hope that the mystery of the "strange metal" phase in underdoped cuprates will be resolved soon. Another unusual feature of some cuprates is their free carrier spectrum. For example, the free spectrum of $\mathrm{Sr}_{2} \mathrm{CuO}_{2} \mathrm{Cl}_{2}$ and of some other materials in the insulating phase has the maxima at the momenta $\mathbf{k}=( \pm \pi / 2, \pm \pi / 2)$ [15]. This fact suggests that the main hopping processes in these systems take place between the next nearest neighbor (NNN) and next NNN sites. In other words, they correspond to an inter-sublattice carrier motion. Since the NNN and next NNN hopping parameters are too small for the oxygen sublattice of the $\mathrm{CuO}_{2}$ planes, it is difficult to believe that the holes move within the oxygen sites, as it is assumed by many researchers [16]. It is also difficult to assume that the free carrier spectrum corresponds to the copper site NNN and next NNN hopping of the Zhang-Rice singlet, formed by an oxygen hole and by one of the copper ions. In fact, the Zhang-Rice singlet states, which move within their magnetic sublattice, are unstable due to the hole frustration with respect to the choice of the axis of the spin quantization and for some other reasons $[17,18]$. In order to describe some of the physical properties of the cuprates with the maximum of the free carrier spectrum at $\mathbf{k}=( \pm \pi / 2, \pm \pi / 2)$, there was proposed a model, in which the holes occupy the sites and move within the copper ion antiferromagnetic sublattice where they were born [17]. It was assumed that the hopping takes place between the NNN and next NNN sites, and the superconducting hole-hole attraction is due to the minimization of the energy of the system, when two holes occupy the nearest sites. In this case, the minimal number of antiferromagnetic bonds is broken [19-23] and similarly to the Hubbard and the $t J$-model cases, the pairing takes places predominantly in the $d$-wave channel [23]. It is important to stress that in order to explain the maximum of the quasi-particle spectrum at $\mathbf{k}=( \pm \pi / 2, \pm \pi / 2)$, one has to neglect the NN hopping, $t=0$, despite the fact that this hopping matrix element is larger than the other hopping parameters. This is due to the fact that the second sublattice hopping does not contribute to the quasi-particle peak. This situation is similar to the exciton motion in an antiferromagnetic system. In the last case, the second sublattice hopping does not contribute to the excitonic quasi-particle peak. It only results in a tail in the absorption spectrum.

We have already studied some of the properties of cuprates in the superconducting and pseudogap phases within the framework of this model. Namely, in [18], we have analyzed the temperaturehole phase diagram of the model in the case of low doping. In this paper, we have obtained the doping dependence of the superconducting critical temperature $T_{\mathrm{c}}$ by solving a system of coupled equations for the Green's function for the Hubbard operators within a generalized mean-field approximation [24]. It was shown that superconductivity in the model arises at finite doping and $T_{\mathrm{c}}$ grows with doping in the underdoped regime. We have also obtained the increase of $T_{\mathrm{c}}$ with doping in the overdoped regime. This result is connected with a simplified approximation of the hole-hole attraction, which was assumed to be doping-independent (see the following section). In order to obtain the decrease of the superconducting critical temperature with doping in the overdoped regime, similar to the spin-polaron model [13], one needs to take into account the doping-dependence of the attraction. Namely, the effective attraction should decay due to the destruction of the antiferromagnetic background with doping increasing. We have also shown that there is an additional pseudogap phase at temperatures above $T_{\mathrm{c}}$ and below another critical temperature $T_{0}$, which also grows with doping increasing in the case of low carrier densities (for a schematic picture, see figure 1). Namely, according to the Emery-Kivelson scenario [25], the Cooper pairs start to form below the temperature $T_{0}$, which is associated with the superconducting mean-field critical temperature $T_{\mathrm{c}}^{M F}$. In the two-dimensional case, the pairs are disordered (the order parameter phases are exponentially ordered) above the condensation temperature $T_{\mathrm{c}}$. This temperature can be associated 


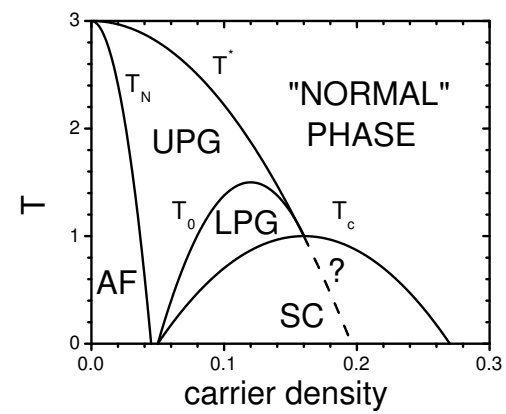

Figure 1. A typical temperature-carrier density phase diagram of the hole-doped cuprates. It consists of the "normal", superconducting (SC), antiferromagnetic (AF), and two pseudogap phase regions: the lower pseudogap (LPG) phase, in which the behavior is affected by both SC and AF spin phase fluctuations, and the upper pseudogap (UPG) phase, in which only the AF spin phase fluctuations are present.

with the Berezinskii-Kosterlitz-Thouless (BKT) critical temperature, below which the phases of the superconducting order parameter are algebraically ordered. This is the only possible critical temperature in the two-dimensional case. The existence of superconducting pseudogap phase with unusual properties at $T_{\mathrm{c}}<T<T_{0}$ was confirmed in some cuprates, where a strong Nernst effect was observed (see, for example [1,26], and a theoretical paper [27]). However, it is well-known that the anomalous properties of cuprates in the underdoped regime take place up to the temperatures much higher than $T_{0}$. In particular, an anomalous pseudogap in the one-hole density of states is observed below a temperature $T^{*} \gg T_{0}$, which is called the pseudogap critical temperature. We present a schematic phase diagram of cuprates in figure 1 , where we distinguish two regions in the pseudogap phase: the superconducting lower pseudogap (LPG) phase and the other one, which we call the upper pseudogap (UPG) phase. The UPG critical temperature $T^{*}$ is a decreasing function of doping. There are some experimental evidences that $T^{*}$ goes below $T_{\mathrm{c}}$ in the overdoped regime and approaches zero at doping $\delta \simeq 0.19$. It is believed by many researchers that the physical properties in the region $T_{0}<T<T^{*}$ are defined by nonsuperconducting processes (for overview, see [1]). One of the popular explanations is based on the idea of the spin singlet formation in a doped two-dimensional antiferromagnet, which corresponds to the resonant valence bond model. Unfortunately, there are no crucial experimental results which confirm the existence of such a state so far. Also, it is known that a non-Fermi liquid temperature dependence of the conductivity in underdoped cuprates takes place up to temperatures of the order of $3000 \mathrm{~K}$. Therefore, as it was suggested by Phil Anderson [28], it shows that probably nonsuperconducting effects are responsible for this phase. Namely, he suggested that the unusual behavior of the spectral function can be explained by a renormalization of the quasiparticle Green's function due to a Gutzwiller projection in a strongly correlated model, which leads to an additional time-dependence of the Green's function and a non-pole-like (cut-like) form of this function in the frequency representation, which corresponds to a non-Fermi-liquid case.

In paper [29], we have studied some of the spectral properties of the model proposed in [17] by taking into account fluctuations of the phases of the superconducting order parameter and the phases of the spins in the antiferromagnetic background. In particular, we have shown that the growth of the Fermi arcs with temperature in underdoped cuprates can be qualitatively explained within the model by taking into account fluctuations of the superconducting $d$-wave order parameter. In this paper, we analyze the effect of the superconducting and spin fluctuations on the structure of one-hole Green's function and the consequent anomalous behavior of the cuprates in the pseudogap phase. In particular, we show that a cut-like structure of the Green's function, qualitatively similar to the one obtained in [28], can be obtained by taking into account these fluctuations. 
The paper is organized as follows. The model and the main equations in the mean-field case are presented in section 2 . In section 3, we extend the problem by taking into account fluctuations of the spins on the antiferromagnetic sublattices and estimate the doping dependence of the UPG critical temperature $T^{*}$. The results for the spectral function and the density of states at different values of temperature are presented in sections 4 and 5 , correspondingly. In addition, in section 5 we show how the anomalous frequency dependence of the conductivity can be obtained from the cut-like Green's function. A summary, a discussion of the results and conclusions are given in section 6 .

\section{Model}

As it was shown in [17], the effective model for the holes in some of the weakly doped cuprates can be written as:

$$
H=\left(\varepsilon_{d}-\mu\right) \sum_{\mathbf{n}} X_{\mathbf{n}}^{2,2}-\frac{1}{2} \sum_{\mathbf{n}, \mathbf{m}} t_{\mathbf{n m}} \cos \frac{\mathbf{Q}_{A F M}(\mathbf{n}-\mathbf{m})}{2} X_{\mathbf{n}}^{2,1 / 2} X_{\mathbf{m}}^{1 / 2,2}-J \sum_{\mathbf{n}, \rho=\mathbf{a}, \mathbf{b}} X_{\mathbf{n}}^{2,2} X_{\mathbf{n}+\rho}^{2,2} .
$$

In the latter equation, $X_{\mathbf{n}}^{2,2}, X_{\mathbf{n}}^{1 / 2,2} X_{\mathbf{n}}^{2,1 / 2}$ are the Hubbard operators for the hole number, the hole annihilation and the hole creation on the site $\mathbf{n}$. The first three terms in the Hamiltonian (1) describe the local hole energy and the NN, and NNN hopping processes, where $\varepsilon_{d}$ and $\mu$ are the hole on-site energy and the chemical potential. The magnetic structure vectors $\mathbf{Q}_{A F M}$ are equal to $( \pm \pi, \pm \pi)$, which corresponds to the antiferromagnetic case. We use the local spin coordinates for the Hubbard operators. The noninteracting part of the hole Hamiltonian describes holes, which move within their sublattices. The free hole dispersion relation is as follows:

$$
\varepsilon(\mathbf{k})=\varepsilon_{d}-4 t_{2} \cos k_{x} \cos k_{y}-2 t_{3}\left(\cos 2 k_{x}+\cos 2 k_{y}\right)-\mu .
$$

The effective hole-hole attraction in the system is described by the last term in equation (1). In fact, the doped holes introduced on the antiferromagnetic lattice, which move within the sublattice they were introduced, lead to a minimal increase of the energy of the system when they sit on the nearest sites [19], since in this case the minimal number of the antiferromagnetic couplings $J$ between the nearest site spins is broken. In this case, two doped holes will always try to occupy NN sites, which results in the effective attraction described by the last term of the Hamiltonian (1). In our calculations, we use the length units such that the lattice constant is equal to one, $a=1$, and choose the energy parameter $\varepsilon_{d}$ to be equal to $4 t_{2}+4 t_{3}$. In this case, the free hole energy is equal to zero at $\mathbf{k}=0$ in the limit of low doping $(\mu \rightarrow 0)$. In order to find the dependence of physical properties of the system on hole concentration $\delta$, we shall use the following equation, which defines $\delta$ in terms of the Hubbard particle number operator:

$$
\delta=\sum_{\mathbf{n}}\left\langle X_{\mathbf{n}}^{2,2}\right\rangle
$$

Recently, we have studied the spectral properties of the model by taking into account superconducting fluctuations [29]. In this paper, we use a similar formalism to study the effect of antiferromagnetic background spin fluctuations on the anomalous spectral and some other properties of the system in the pseudogap phase at $T>T_{\mathrm{c}}$. Although we are interested mainly in the spin fluctuation effects, we shall consider the general case assuming that the superconducting pairing can also take place, which corresponds to the temperature interval $T_{\mathrm{c}}<T<T_{0}$. At higher temperatures, i.e. at $T_{0}<T<T^{*}$, we shall use the same equations by putting the superconducting gap to be equal to zero in our calculations.

Then, in order to study the properties of the system described by the Hamiltonian (1), it is convenient to introduce generalized Nambu-Hubbard hole operators

$$
\Psi_{\mathbf{n}}(t)=\left(\begin{array}{c}
X_{\mathbf{n}}^{2,1 / 2}(t) \\
X_{\mathbf{n}}^{1 / 2,2}(t)
\end{array}\right), \quad \Psi_{\mathbf{n}}^{\dagger}(t)=\left(X_{\mathbf{n}}^{1 / 2,2}(t), X_{\mathbf{n}}^{2,1 / 2}(t)\right)
$$


where $\mathbf{n}$ is the lattice site and $t$ is time, and to calculate the time-ordered Green's function

$$
\hat{G}_{\mathbf{n m}}\left(t, t^{\prime}\right)=-\mathrm{i}\left\langle T\left(\Psi_{\mathbf{n}}(t) \Psi_{\mathbf{m}}^{\dagger}\left(t^{\prime}\right)\right)\right\rangle \text {. }
$$

This function satisfies the following equation:

$$
\mathrm{i} \frac{\partial}{\partial t} \hat{G}_{\mathbf{n m}}\left(t, t^{\prime}\right)=\delta\left(t-t^{\prime}\right) \delta_{\mathbf{n} \mathbf{m}} \hat{I}+\left\langle T\left[\Psi_{\mathbf{n}}(t), H\right] \Psi_{\mathbf{m}}^{\dagger}\left(t^{\prime}\right)\right\rangle
$$

where $\hat{I}$ is a diagonal $2 \times 2$-matrix with the nonzero elements equal to $\left\langle X_{\mathbf{n}}^{1 / 2,1 / 2}(t)+X_{\mathbf{n}}^{2,2}(t)\right\rangle$. In the case of low doping, $\hat{I} \simeq \hat{1}$. In order to solve equation (6), it is convenient to approximate its last term by a generalized mean-field theory expression:

$$
\left\langle T\left[\Psi_{\mathbf{n}}, H\right] \Psi_{\mathbf{m}}^{\dagger}\right\rangle(\omega) \simeq \sum_{\mathbf{l}} \hat{E}_{\mathbf{n} \mathbf{l}} \hat{G}_{\mathbf{l m}}(\omega),
$$

where

$$
\hat{E}_{\mathbf{n m}}=\left\langle\left\{\left[\Psi_{\mathbf{n}}, H\right], \Psi_{\mathbf{m}}^{\dagger}\right\}\right\rangle
$$

is the energy matrix (for details see, e.g., [24]). In this approximation, one neglects the dynamical corrections to the self-energy, which can be systematically taken into account. For example, in [12] the authors considered these corrections in the case of the $t J$-model by using a similar formalism. In order to find the Green's function, one needs to calculate the elements of the energy matrix $\hat{E}_{\mathbf{n l}}$, which depend on different correlation functions, in particular, on the superconducting gap function, which can be found by using the fluctuation-dissipation theorem. Assuming that the superconducting pairing takes place in the $d$-wave channel and introducing the relevant gap function:

$$
\Delta_{d}(\mathbf{k})=-4 J \sum_{\mathbf{q}} \gamma_{d}(\mathbf{k}) \gamma_{d}(\mathbf{q})\left\langle X_{-\mathbf{q}}^{2,1 / 2} X_{\mathbf{q}}^{2,1 / 2}\right\rangle \equiv \Delta_{d} \gamma_{d}(\mathbf{k})
$$

where $\gamma_{d}(\mathbf{k})=\cos \left(k_{x}\right)-\cos \left(k_{y}\right)$ is a $d$-wave structure factor and the $X$-operators belong to different sublattices, one can get the following approximate expression for the Green's function in the frequency-momentum representation:

$$
G(\omega, \mathbf{k})=\frac{1}{\omega+\varepsilon(\mathbf{k}) \tau_{z}+\mathrm{i} \Delta(\mathbf{k}) \tau_{y}}
$$

where $\hat{\tau}_{y}$ and $\hat{\tau}_{z}$ are the Pauli matrices (for details, see [18]).

In order to find the doping and temperature dependencies of the superconducting gap parameter $\Delta_{d}$, one needs to derive and solve the system of equations for $\Delta_{d}$ and the chemical potential $\mu$. These equations follow from the definitions (3), (9) and the self-consistency conditions, which follow from the fluctuation-dissipation theorem:

$$
\begin{aligned}
1 & =4 J \sum_{\mathbf{q}} \gamma_{d}^{2}(\mathbf{q}) \tanh \left(\frac{\sqrt{\varepsilon^{2}(\mathbf{q})+\Delta_{d}^{2} \gamma_{d}^{2}(\mathbf{q})}}{2 T}\right) \frac{1}{\sqrt{\varepsilon^{2}(\mathbf{q})+\Delta_{d}^{2} \gamma_{d}^{2}(\mathbf{q})}}, \\
\delta & =\sum_{\mathbf{q}}\left[1+\tanh \left(\frac{\sqrt{\varepsilon^{2}(\mathbf{q})+\Delta_{d}^{2} \gamma_{d}^{2}(\mathbf{q})}}{2 T}\right) \frac{\varepsilon(\mathbf{q})}{\sqrt{\varepsilon^{2}(\mathbf{q})+\Delta_{d}^{2} \gamma_{d}^{2}(\mathbf{q})}}\right]
\end{aligned}
$$

(see [18] for details). The solution of these equations at $\Delta_{d}=0$ gives the doping dependence of the mean-field critical temperature $T_{\mathrm{c}}^{M F}$, or the LPG critical temperature $T_{0}$. According to the EmeryKivelson scenario, the real superconducting critical temperature $T_{\mathrm{c}}<T_{\mathrm{c}}$ in the two-dimensional case corresponds to the BKT temperature, below which the phases of the order parameter become algebraically ordered (for over-review see, for example, [30]). We studied the doping dependence of this temperature in [18]. In this paper, we shall mainly concentrate on the spectral properties of the model in the pseudogap phase at $T>T_{\mathrm{c}}$ by taking into account superconducting fluctuations and spin fluctuations of the antiferromagnetic background. 


\section{Spin fluctuations}

In order to describe the UPG region, we assume that, in analogy with the superconducting order parameter fluctuations, the physics in the temperature range $T_{0}<T<T^{*}$ is governed by fluctuations of spin phases. Supposing that the antiferromagnetic copper oxide spin Hamiltonian is described by the XY-model, it is easy to obtain an analogous critical temperature for the spin subsystem. Therefore, we associate $T^{*}$ with the temperature of the BKT transition for spins. This temperature can be also estimated from the following equation:

$$
T^{*}=\frac{\pi}{2} \mathcal{J}_{\text {spin }}\left(\mu, T^{*}\right)
$$

where the spin stiffness $\mathcal{J}_{\text {spin }}$ is the coefficient in front of the quadratic term of the spin phase gradients in the effective action for the spin phase $\varphi_{\mathbf{n}}=\mathbf{Q}_{A F M} \mathbf{n}$ differences:

$$
\Omega=\frac{\mathcal{K}_{\text {spin }}}{2} \int \mathrm{d}^{2} r \varphi \partial_{t}^{2} \varphi+\frac{\mathcal{J}_{\text {spin }}}{2} \int \mathrm{d}^{2} r(\nabla \varphi)^{2}
$$

It is equal to

$$
\mathcal{J}_{\text {spin }}=\frac{1}{2} \int \frac{\mathrm{d}^{2} k}{(2 \pi)^{2}} \tanh \left(\frac{\sqrt{\bar{\varepsilon}^{2}(\mathbf{k})+\Delta_{d}^{2}(\mathbf{k})}}{2 T}\right) \frac{\bar{\varepsilon}^{2}(\mathbf{k})}{\sqrt{\bar{\varepsilon}(\mathbf{k})^{2}+\Delta_{d}^{2}(\mathbf{k})}}
$$

where $\bar{\varepsilon}(\mathbf{k})=\varepsilon(\mathbf{k})-4 J \delta+4 t_{1} \delta\left(\cos k_{x}+\cos k_{y}\right)$ (see [29]). As it was mentioned above, doping leads to a gradual destruction of the antiferromagnetic order and to a hole hopping between NN sites, which belong to different sublattices. We take this process into account by adding the dopingdependent term $4 t_{1} \delta\left(\cos k_{x}+\cos k_{y}\right)$ to the free spectrum (2). The doping dependence of $T^{*}$ can be found from equation (13) at $\Delta_{d}=0$. In this case, equation (13) has the following form:

$$
T^{*}=\frac{\pi}{4} \int \frac{\mathrm{d}^{2} k}{(2 \pi)^{2}} \tanh \left(\frac{\bar{\varepsilon}(\mathbf{k})}{2 T^{*}}\right) \bar{\varepsilon}(\mathbf{k}) .
$$

The exact solution of equation (16) shows that $T^{*}$ is a decreasing function of doping. One can estimate the analytical dependence $T^{*}(\delta)$ from equation (16) by taking into account that at low doping $T^{*} \gg|\bar{\varepsilon}(\mathbf{k})|$. Therefore, in this case:

$$
T^{*} \simeq \sqrt{\frac{\pi}{8} \int \frac{\mathrm{d}^{2} k}{(2 \pi)^{2}} \bar{\varepsilon}^{2}(\mathbf{k})} \simeq T_{\max }^{*}(1-\beta \delta),
$$

where $T_{\max }^{*}=\left.\sqrt{\frac{\pi}{8} \int \frac{\mathrm{d}^{2} k}{(2 \pi)^{2}} \bar{\varepsilon}^{2}(\mathbf{k})}\right|_{\delta=0}$ and $\beta=4 \sqrt{\pi / 2} t_{1} \int \frac{\mathrm{d}^{2} k}{(2 \pi)^{2}} \varepsilon(\mathbf{k})\left(\cos k_{x}+\cos k_{y}\right)$.

\section{Spectral function}

In order to study the spectral function in the case when the spin phase fluctuations are taken into account, it is convenient to write down the spin functions explicitly in the laboratory system of coordinates:

$$
\left|\sigma_{\mathbf{n}}\right\rangle=|\sigma\rangle \cos \left(\varphi_{\mathbf{n}} / 2\right)+2 \sigma|\sigma\rangle \sin \left(\varphi_{\mathbf{n}} / 2\right)
$$

where $\varphi_{\mathbf{n}}$ is the angle between the directions of the laboratory and local systems of coordinates. For simplicity, we assume that the spins lie in the plane of the system and their phase fluctuations are small. In this case, one can get the following result for the Green's function:

$$
G_{\alpha \beta}(x)=\mathcal{G}_{\alpha \beta}(x) \mathcal{D}^{\operatorname{spin}}(x),
$$

where $\mathcal{G}_{\alpha \beta}(x)$ is the Green's function of the Nambu spinors and

$$
\mathcal{D}^{\operatorname{spin}}(x)=\mathrm{e}^{-\langle\varphi(x) \varphi(0)\rangle / 4}
$$


is the correlator of the spin phases (for details, see $[29,30,32]$ ). The phase correlators in the last two expressions can be easily obtained from the effective action for the spin phases. Namely,

$$
D^{\operatorname{spin}}(\tau, \mathbf{r})=\exp \left[-\frac{T}{4} \sum_{n=-\infty}^{\infty} \int \frac{q \mathrm{~d} q \mathrm{~d} \varphi}{(2 \pi)^{2}} \frac{1-\cos (q r \cos \varphi) \cos \left(\Omega_{n} \tau\right)}{\mathcal{J}_{\text {spin }} q^{2}+\mathcal{K}_{\text {spin }} \Omega_{n}^{2}}\right]
$$

where $\mathcal{J}_{\text {spin }}$ and $\mathcal{K}_{\text {spin }}$ are the coefficients in front of the gradient and time derivative terms in the effective action (14). One can use these exact expressions to calculate the spin correlation functions. However, in order to study the qualitative behavior of the spectral function of the system, it is enough to approximate the correlation function by

$$
D_{\text {spin }}(x)=\left[\theta\left(T^{*}-T\right)+\theta\left(T-T^{*}\right) \exp \left(-r / \xi_{\text {spin }}(T)\right)\right] \mathrm{e}^{-\Gamma_{\text {spin }} t}\left(r / r_{\text {spin }}\right)^{-\alpha_{\text {spin }}},
$$

where $T^{*}$ is the spin BKT temperature and $\alpha_{\text {spin }}, \xi_{\text {spin }}, r_{\text {spin }}$ and $\Gamma$ are doping- and temperaturedependent parameters. In principle, one also needs to include superconducting fluctuations in order to describe the properties of the system at $T_{\mathrm{c}}<T<T_{0}$. In this case, $D^{\text {spin }}$ should be multiplied by the corresponding superconducting function $D^{\mathrm{SC}}$ with the space-time dependence similar to equation (20). We assume that $D^{\text {spin }}$ describes the total effect of the spin and superconducting fluctuations and use the corresponding parameter notations $\alpha, \xi, r$ and $\Gamma$ instead of the ones used in equation (20). The values of the parameters can be estimated from experiments (see below).

In the frequency-momentum representation, the Green's function has the following form:

$$
G\left(\mathrm{i} \omega_{n}, \mathbf{k}\right)=-T \sum_{m} \int \mathrm{d} \mathbf{q} \frac{\mathrm{i} \omega_{m}+\tau_{3} \varepsilon(\mathbf{q})}{\omega_{n}^{2}+\varepsilon^{2}(\mathbf{q})+\Delta_{d}^{2}(\mathbf{q})} \mathcal{D}^{\text {spin }}\left(\mathbf{k}-\mathbf{q}, \omega_{n}-\omega_{m}\right),
$$

where the Fourier transform of the correlator of the phase fluctuations $\mathcal{D}^{\text {spin }}\left(\mathbf{k}-\mathbf{q}, \omega_{n}-\omega_{m}\right)$ can be found from equation (20). In particular, at $T<T^{*}$ :

$$
D^{\text {spin }}\left(\mathrm{i} \Omega_{n}, \mathbf{q}\right)=A \frac{1}{\left[\mathbf{q}^{2}+\xi^{-2}(T)\right]^{\alpha}} \frac{\Gamma}{\omega_{n}^{2}+\Gamma^{2}},
$$

where $A$ is a parameter. We shall use this expression to analyze the spectral properties of the system. For simplicity, we assume that the inverse time correlation length $\Gamma$ is proportional to temperature and put $\Gamma=0.1 T / \bar{T}$, where $\bar{T} \sim T^{*}$.

The spectral properties of the holes can be studied by making the analytical continuation $\mathrm{i} \omega_{n} \rightarrow \omega+\mathrm{i} \eta(\eta \rightarrow+0)$ and extracting the imaginary part of the Green's function

$$
A(\omega, \mathbf{k})=-(\mathrm{i} / \pi) \operatorname{Im} G(\omega, \mathbf{k})
$$

In this case, by using equations (21)-(23) and performing one frequency integration, one can get the following expression for the spectral function:

$$
\begin{aligned}
A(\omega, \mathbf{k})= & A \int \mathrm{d} \mathbf{q}\left\{\left[1+\frac{\xi(\mathbf{q})}{E(\mathbf{q})}\right] \frac{\Gamma}{(\omega-E(\mathbf{q}))^{2}+\Gamma^{2}}+\left[1-\frac{\xi(\mathbf{q})}{E(\mathbf{q})}\right] \frac{\Gamma}{(\omega+E(\mathbf{q}))^{2}+\Gamma^{2}}\right\} \\
& \times \frac{1}{\left[(\mathbf{k}-\mathbf{q})^{2}+\xi^{-2}(T)\right]^{\alpha}},
\end{aligned}
$$

where $A$ can be most easily found by using the Green's function zeroth spectral moment sum rule. Let us get an approximate expression for the spectral function in the case of $\Delta_{d}=0$ and positive frequencies. In this case, the last term in the figure brackets can be neglected, and one gets

$$
A(\omega, \mathbf{k}) \simeq \bar{A}(\omega) \int \mathrm{d} \varphi_{q} \frac{1}{\left[\left(\mathbf{k}^{2}-2 k \sqrt{2 m^{*}(\mu+\omega)} \cos \left(\varphi_{q}\right)+2 m^{*}(\mu+\omega)+\xi^{-2}(T)\right]^{\alpha}\right.},
$$

where

$$
\bar{A}(\omega)=A m^{*} \int_{0}^{\infty} \mathrm{d} \xi[1+\operatorname{sign}(\xi-\mu)] \frac{\Gamma}{(\omega-\xi)^{2}+\Gamma^{2}} \simeq 2 m^{*} A\left[\frac{\pi}{2}+\arctan (\omega / \Gamma)\right] .
$$


As it follows from equation (25), the Green's function has a cut-like form, contrary to the Fermiliquid pole-like case. Such a dependence is caused by the presence of the spin correlation function $\mathcal{D}^{\text {spin }}\left(\mathbf{k}-\mathbf{q}, \omega_{n}-\omega_{m}\right)$ in equation $(21)$, which smoothes out the $\delta$-function peaks of the spectral function that come from the denominator of the fermion Green's function. Therefore, the quasiparticle residue $Z$, which can be defined as the coefficient in front of the Fermi quasi-particle spectral function $\delta$-peak, is equal to zero. This means that the system is in a non-Fermi-liquid regime. This result is qualitatively similar to the result recently obtained by P.W. Anderson in the case of a strongly correlated model [28] and used to describe the spectral function in $\mathrm{Bi}_{2} \mathrm{Sr}_{2} \mathrm{CaCu}_{2} \mathrm{O}_{8+\delta}$ in $[33]$ :

$$
A(\omega, \mathbf{k})=f\left(\frac{\omega}{T}\right) \frac{\sin \left[(1-p)\left(\pi / 2-\tan ^{-1}\left[\left(\omega-v_{\mathrm{F}} k\right) / \Gamma\right]\right)\right]}{\left[\left(\omega-v_{\mathrm{F}} k\right)^{2}+\Gamma^{2}\right]^{(1-p) / 2}},
$$

where $f(\omega / T)$ is the Fermi function and $\mathrm{p}$ and $\Gamma$ are parameters in the effective Green's function:

$$
G(t, \mathbf{x})=\frac{t^{-p} \mathrm{e}^{-\Gamma t}}{|\mathbf{x}|-v_{\mathrm{F}} t}
$$

In particular, the prefactor $t^{-p}$ comes from the contribution of the Gutzwiller projection on the single occupied states of the strongly correlated system. It was estimated that $p \simeq 0.12$ and $\Gamma=A T+B\left(k-k_{\mathrm{F}}\right)^{2}$ in the momentum space, where $A$ and $B$ are constants.

In fact, in our case we can get an approximate cut-like expression for the spectral function by putting $\varphi_{q}=0$ in the expression under the integral in equation (25) and integrating over the angle $\varphi_{q}$. This can be done since the momentum angle region around $\varphi_{q}=0$ gives the largest contribution into the integral. In this case,

$$
A(\omega, \mathbf{k}) \simeq 4 \pi m^{*} A\left[\frac{\pi}{2}+\arctan (\omega / \Gamma)\right] \frac{1}{\left[\left(k-\sqrt{2 m^{*}(\mu+\omega)}\right)^{2}+\xi^{-2}(T)\right]^{\alpha}} .
$$

As it follows from this equation, the spectral function has the maximum at $\omega=\xi(\mathbf{k})=$ $k^{2} /\left(2 m^{*}\right)$, similar to the free hole case (we put $\mu=0$ for simplicity), but this function is a smooth function, different from the delta-function. Our numerical evaluation of the integral in equation (25) show that the approximation (28) is correct only at large values of $\alpha$ (figure 2). In the case of small $\alpha$, the $\omega[\xi(\mathbf{k})]$-dependence for the spectral function maximum is linear at large $\xi(\mathbf{k})$. In this case, the curve begins at finite value of $|\mathbf{k}|$, which decreases with $\alpha$ increasing.

From equation (28), one can find an approximate expression for the spectral function at $\omega=0$ :

$$
A(\omega=0, \mathbf{k}) \simeq 2 \pi^{2} m^{*} A \frac{1}{\left[\left(k-k_{\mathrm{F}}\right)^{2}+\xi^{-2}(T)\right]^{\alpha}} .
$$

As it follows from this equation, the spectral weight at the Fermi level is defined by $\xi$ and $\alpha$.

\section{Density of states}

The density of states can be obtained from equation (24) by using the standard expression

$$
N(\omega)=\int \frac{\mathrm{d}^{2} k}{(2 \pi)^{2}} A(\omega, \mathbf{k})
$$

Similar to the previous section, one can show that the system demonstrates a finite DOS at zero frequency and it is defined by the parameters $\xi$ and $\alpha$.

One can estimate the DOS weight at $\omega=0$ from equation (29):

$$
\begin{aligned}
N(\omega=0) & \simeq 2 \pi^{2} m^{*} A \int \frac{d^{2} k}{(2 \pi)^{2}} \frac{1}{\left[k^{2}+\xi^{-2}(T)\right]^{\alpha}} \\
& \simeq \frac{\pi m^{*} A}{2(1-\alpha) \xi^{2(1-\alpha)}}\left[\left(2 m^{*} W \xi^{2}(T)+1\right)^{1-\alpha}-1\right] .
\end{aligned}
$$




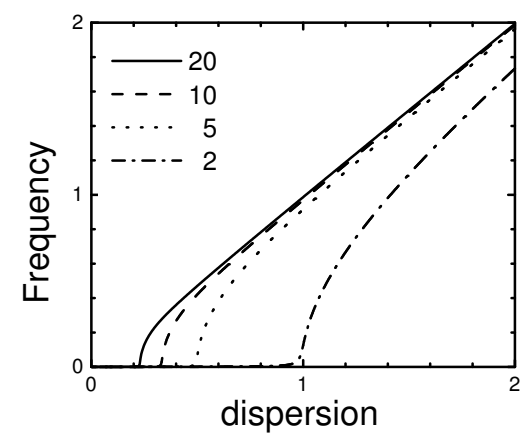

Figure 2. The spectral function peak frequency $\omega$-dispersion $\xi(\mathbf{k})$ curve at different values of $\alpha$. This curve approaches the free fermion curve $\omega=\xi(\mathbf{k})$ as $\alpha$ increases.

From equations (28), (29) and (31) one can estimate the values of phenomenological parameters $\xi, \alpha$ and $\Gamma$ by comparing theoretical results with experimental data. In particular, one can get that $\Gamma \sim T$ and $\xi$ is a weakly-dependent function of temperature for a wide temperature range above $T_{0}$.

To conclude this section, we would like to demonstrate how some of the non-Fermi-liquid properties could result from the cut-like structure of the Green's function by using the anomalous conductivity as an example. One can roughly estimate the conductivity to be proportional to the quasiparticle life-time $\tau(\mathbf{k}, \omega)$ at $|\mathbf{k}|=k_{\mathrm{F}}$. This quantity can be estimated to be inversely proportional to the imaginary part of the one-hole self-energy, i.e. $\tau\left(k_{\mathrm{F}}, \omega\right) \sim 1 / \operatorname{Im} \Sigma\left(k_{F}, \omega\right)$. On the other hand, since

$$
A(\mathbf{k}, \omega)=\frac{\operatorname{Im} \Sigma(\mathbf{k}, \omega)}{\left(\mathbf{k}^{2} / 2 m^{*}-\mu\right)^{2}+\operatorname{Im} \Sigma^{2}(\mathbf{k}, \omega)},
$$

where we have neglected the real part of the self-energy, one immediately gets $\tau\left(k_{\mathrm{F}}, \omega\right) \sim A\left(k_{F}, \omega\right)$. The frequency dependence of this quantity at different values of $\alpha$ is presented in figure 3 , where we have subtracted the frequency-independent part from the spectral function. As it follows from this figure, at low and moderate frequencies the dependence of the conductivity on frequency can be approximated by $\sigma \sim \omega^{b}$, where $b$ is a parameter. As it follows from equation (25), at small frequencies

$$
A\left(k_{\mathrm{F}}, \omega\right)-A\left(k_{\mathrm{F}}, \omega\right) \sim \frac{\alpha^{2} \xi\left(k_{\mathrm{F}}\right) / \xi(T)-\alpha}{\left(\xi\left(k_{\mathrm{F}}\right) / \xi(T)+1\right)^{\alpha+2}} \omega .
$$

Such a dependence obtained by using a rather rough approximation already indicates that the cut-like form of the Green's function can result in a non-Fermi-liquid behavior of the system.

In order to make a comparison with experiments for conductivity and other quantities in the pseudogap phase, one needs to more accurately take into account different properties of the materials, like the band structure, the antiferromagnetic spin coupling, which defines $J$ and others. Such a comparison is also necessary in order to estimate the values of the phenomenological parameters for the spin fluctuation correlation function (20).

\section{Conclusions}

In this paper, we have considered the spectral properties of a phenomenological model of HTSC in the underdoped regime by taking into account fluctuations of the phases in the AF spin background. Namely, we have considered the temperature evolutions of the spectral function and of the density of states. By studying the spectral function, we have shown that its temperature dependence in the case of HTSCs can be qualitatively described by this model in the case of a proper choice of the decoherence time correlation length and other parameters for the spin angle correlation function. These parameters can be taken from experiments and they are directly connected 


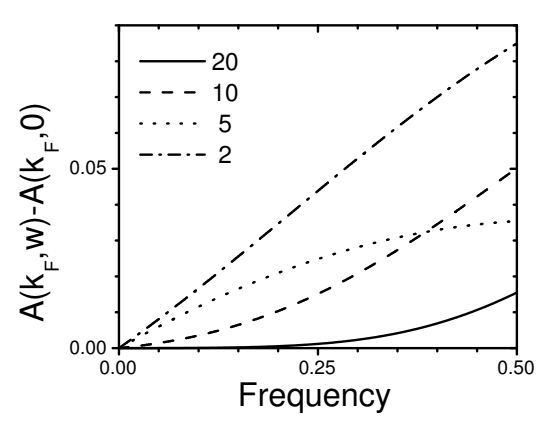

Figure 3. The leading frequency-dependent term of the spectral function (in arbitrary units) at small $\omega, k=k_{\mathrm{F}}$ and different values of $\alpha$. This term is proportional to $A\left(k_{\mathrm{F}}, \omega\right)-A\left(k_{\mathrm{F}}, 0\right)$. The frequency $\omega$ is given in units of $1 /\left(2 m^{*} \xi^{2}\right)$.

to the microscopic model parameters. Similarly, we have derived and analyzed the expression for the density of states. Finally, we have shown that the spin fluctuations can be responsible for the anomalous behavior of the conductivity in the underdoped regime. We have compared our result for the Green's function with the expression proposed in [28], and have shown that both models can describe anomalous properties of underdoped cuprates without using exotic models, like the marginal Fermi-liquid etc. The validity of the model studied in this paper to describe all the properties of cuprates in the pseudogap phase can be tested by taking into account different experimental phenomena, which requires a further investigation.

\section{Acknowledgements}

It is a great pleasure for us to contribute with this paper to a special issue of Condensed Matter Physics on the occasion of the 70th anniversary of a well-known Ukrainian theoretical physicist, a Corresponding Member of The National Academy of Sciences of Ukraine, Professor Ihor V. Stasyuk, who contributed both in the fields of the electronic properties of strongly correlated systems and the theory of superconductivity, including the high- $T_{\mathrm{c}}$ case.

V.M.L. would like to thank P.W. Anderson for a beneficial communication. V.T. acknowledges support by the National Science Foundation under grant number DMR-0553485.

\section{References}

1. Lee P.A., Nagaosa N., Wen X.-G., Rev. Mod. Phys., 2006, 78, 17.

2. Shen Z.-X., Dessau D.S., Phys. Rep., 1995, 253, 1.

3. Ding H., Yokota T., Campuzano J.C., et al., Nature (London), 1996, 382, 51.

4. Loeser A.G., Shen Z.-X., Dessau D.S., Science, 1996, 273, 325.

5. Macridin A., Moritz B., Jarrell M., Maier Th. Preprint arXiv:cond-mat/0611067, 2006.

6. Anderson P.W., Science, 1987, 235, 1196.

7. Zhang F.C., Rice T.M., Phys. Rev. B, 1988, 37, 3759.

8. Kane C.L., Lee P.A., Read. N., Phys. Rev. B, 1989, 39, 6880.

9. Martinez G., Horsch P., Phys. Rev. B, 1991, 44, 317.

10. Bensimon D., Zeyher R. Preprint arXiv:cond-mat/0603585, 2006.

11. Haule K., Kotliar G. Preprint arXiv:cond-mat/0605149, 2006.

12. Plakida N.M., Oudovenko V.S., Phys. Rev. B, 1999, 59, 11949.

13. Plakida N.M., Oudovenko V.S., Horsch P., Liechtenstein A.I., Phys. Rev. B, 1997, 55, R11997.

14. Jaklic J., Prelovsek P., Phys. Rev. B, 1997, 55, R7307.

15. Damascelli A., Hussain Z., Shen Z.-X., Rev. Mod. Phys., 2003, 75, 473.

16. Emery V.J., Phys. Rev. Lett., 1987, 58, 2794.

17. Loktev V.M., Low Temp. Phys., 2005, 31, 490. 
18. Loktev V.M., Turkowski V.M., Phys. Rev. B, 2007, 75, 224513.

19. Trugman S.A., Phys. Rev. B, 1988, 37, 1597.

20. Wrobel P., Eder R., Micnas R., J. Phys.: Condens. Matter, 2003,15, 2755.

21. Wrobel P., Eder R., Fulde P., J. Phys.: Condens. Matter, 2003, 15, 6599.

22. Maciag A., Wrobel P., Phys. Status Solidi B, 2006, 243, 512.

23. Scalapino D.J., Trugman S.A., Philos. Mag. B, 1996, 74, 607.

24. Mancini F., Avella A., Adv. Phys., 2004, 53, 537.

25. Emery V.J., Kivelson S., Nature (London), 1995, 374, 434.

26. Wang Ya., Li Lu, Ong N.P., Phys. Rev. B, 2006, 73, 024510.

27. Anderson P.W. Preprint arXiv:cond-mat/0701042, 2007.

28. Anderson P.W., Nat. Phys., 2006, 2, 626.

29. Loktev V.M., Turkowski V., submitted to Phys. Rev. B.

30. Loktev V.M., Quick R.M., Sharapov S.G., Phys. Rep., 2001, 349, 2.

31. Sharapov S.G., Beck H., Loktev V.M., Phys. Rev. B, 2001, 64, 134519.

32. Patashinskii A.Z., Pokrovskii V.L. Fluctuation Theory of Phase Transitions. Pergamon Press, Oxford, 1979.

33. Casey P.A., Koralek J.D., Plumb N.C., Dessau D.S., Anderson P.W., Nat. Phys., 2008, 4, 210.

\title{
Спінові флуктуації та поведінка типу “дивного металу” у слабо легованому антиферомагнетику
}

\author{
В.М.Локтєв ${ }^{1}$, В.Турковський ${ }^{2}$ \\ ${ }^{1}$ Інститут теоретичної фізики ім. М.М. Боголюбова НАН України, \\ 03680 Київ, вул. Метрологічна, 14-б \\ 2 Факультет фізики та астрономії, університет Міссурі, Колумбія, 65211 Міссурі, США \\ Отримано 16 травня 2008 р., в остаточному вигляді - 18 червня 2008 р.
}

\begin{abstract}
Проаналізовано спектральні властивості феноменологічної моделі для слабо легованого двовимірного антиферомагнетика, в якому носії рухаються у саме тій з двох підграток, куди вони були введені. Таке обмеження приводить до спектрів вільних носіїв з максимумами при $\mathbf{k}=( \pm \pi / 2, \pm \pi / 2)$, що спостерігаються у деяких купратах. Спектральні властивості моделі досліджено шляхом врахування флуктуацій спінів відносно антиферомагнітного фону. Показано, що такі флуктуації ведуть до безполюсної структури однодіркових функцій Ґріна, а також ці флуктуації можуть відповідати за певні аномальні властивості недолегованих купратів типу “дивного металу” у ненадпровідному режимі.
\end{abstract}

Ключові слова: купратні надпровідники, недолегований режим, псевдощілина

PACS: 74.20.-z, 74.20.Fg, 74.20.Rp, 74.72.-h 
